\title{
Introduction to Quantum Field
} Theory in $C^{4}$ space-time

\section{D.Mastoridis -1}

Amaliados 17, Athens, Greece, P.O.Box 11523, d.mastoridis@prv.ypeka.gr

$$
\text { K.Kalogirou }-2
$$

Amaliados 17, Athens, Greece, P.O.Box 11523, k.kalogirou@prv.ypeka.gr keywords:unified theories;complex space-time;gauge theories;particle theory;Higg's boson;geoemetric unification 


\begin{abstract}
We explore the possibility to find the usual quantum theories, within the formulation of a classic theory of mechanics in $C^{4}$. Specifically, by releasing the end-point of the integral of the action derived in $C^{4}$, we derive the dynamic path length of the geodesic equation in $C^{4}$. In the flat case, the derived Hamilton-Jacobi equations, were identified as the usual Klein-Gordon equation, where the complex functional action $S\left(z_{i}\right)$, is identified as the usual complex scalar field $\varphi$. Afterwards, we study the energy-momentum 4-d complex vector, in order to re-establish the usual covariant derivative of gauge theories.
\end{abstract}




\section{Introduction}

This is the third paper of a series of papers [?] [?] [?] [?] [?], concerning a physical theory in an extended $C^{4}$ space-time. We have derived in the first paper [?] the geodesic equations firstly in the symplectic $R^{8}$ and after in the embedded usual space-time in it. It is natural to investigate what will happen in the case of a free endpoint theory which will help us to derive the Hamilton-Jacobi equations. We will show that this effort will help us to define what a quantum theory actually represents.

\section{Lagrangian formalism}

Let us remind us some preliminaries on the subject of free endpoint as they exist in the context of Lagrangian mechanics. Let us consider an action with respect to a Lagrangian that describes a physical system as

$$
S=\int_{a}^{b} L(x, \dot{x}, t) d t
$$

where a,b fixed points of the usual 4-d space-time. The principle of least action $\delta S=0$ leads to the usual Euler-Lagrange equations. Now, if we change a little bit the "history" by varying the endpoint by $\Delta \mathrm{x}=\delta x-\dot{x} \delta t$ (for more details see "Gravitation" of C.Missner, K. Thorne, J.Wheeler) the variation of the action becomes

$$
\begin{gathered}
\delta S=L(x, \dot{x}, t) \delta t+\int_{a}^{b+\Delta \mathbf{x}} \delta L d t \\
=L \delta+\int_{a}^{b+\Delta \mathbf{x}}\left(\frac{\partial L}{\partial \dot{x}} \delta \dot{x}+\frac{\partial L}{\partial x} \delta x\right) d t \\
=L \delta t+\frac{\partial L}{\partial \dot{x}} \Delta \mathbf{x}+\int_{a}^{b+\Delta \mathbf{x}}\left(\frac{\partial L}{\partial x}-\frac{d}{d t} \frac{\partial L}{\partial \dot{x}}\right) \delta x d t
\end{gathered}
$$

the quantity inside the integral is zero, because it represents the usual geodesic equation. By substituting $\Delta \mathrm{x}$ we have

$$
\delta S=\frac{\partial L}{\partial \dot{x}} \delta x-\left(\dot{x} \frac{\partial L}{\partial \dot{x}}-L\right) \delta t
$$


this way the dispersion relation is

$$
\begin{gathered}
\delta S=p \delta x-E \delta t \\
p=\frac{\partial L}{\partial \dot{x}} \quad E=x \frac{\partial L}{\partial \dot{x}}-L
\end{gathered}
$$

the second term is the usual Hamiltonian of the system (Lagrange transformation) which can be read also as

$$
-\frac{\partial S}{\partial t}=H\left(\frac{\partial S}{\partial x}, x, t\right)
$$

In this spirit, if we begin with the ordinary action of a charge in a gravitational field as an application, we begin with an action of the form

$$
S=-\int_{a}^{b} m c d s-\frac{q}{c} A_{i} d x^{i}
$$

The term that was vanished at the limits is

$$
m c u_{i}+\frac{q}{c} A_{i}
$$

But, if we vary the endpoint this term does not vanish( the geodesic equation still holds) and leads to the dispersion relation

$$
\delta S=-\left(m c u_{i}+\frac{q}{c} A_{i}\right) \delta x^{i}
$$

and afterwards to the well known relation

$$
\frac{\partial S}{\partial x^{i}}=m c u_{i}+\frac{q}{c} A_{i}=p_{i}+\frac{q}{c} A_{i}
$$


this relation is the starting point to form the covariant derivative of electrodynamics in quantum field theories. The quantity $\frac{\partial S}{\partial x^{i}}$ is the four vector of the generalised momentum of the particle of charge $\mathrm{q}$, where $p_{i}$ is the ordinary momentum as it exists in the context of mechanics. The most surprising element is that the term $\frac{q}{c} A_{i}$ defines also a momentum term, that is formed directly from the electromagnetic field. Adding the usual momentum $p_{i}$ with the "electromagnetic momentum" we form a generalised momentum. But, through our consideration we have seen in [?] that the electromagnetic field (preferably the generalised $K_{\mu}$ field) is not just an ad-hoc quantity added to the usual geometry of the usual space-time, but rather a geometric term introduced by the antisymmetric tensor $I_{\mu \nu}$. If we put the pieces into order, and start with the elementary length of the extended space-time in the symplectic $R^{8}$

$$
d s^{2}=g_{i j} d x^{i} d x^{j}+g_{i j} d y^{i} d y^{j}+I_{i j}\left(d x^{i} d y^{j}-d y^{j} d x^{i}\right)
$$

and the variation of the action

$$
\delta S=\delta \int\left(g_{i j} u^{i} u^{j}+g_{i j} v^{i} v^{j}+2 I_{i j} u^{i} v^{j}\right) d s
$$

the free endpoint will lead us to the pair of equations

$$
\begin{aligned}
& \frac{\partial S}{\partial x^{i}}=g_{i j} u^{i}+I_{i j} v^{i} \\
& \frac{\partial S}{\partial y^{i}}=g_{i j} v^{i}-I_{i j} u^{i}
\end{aligned}
$$

or in matrix form

$$
\left(\begin{array}{c}
\frac{\partial S}{\partial x^{i}} \\
\frac{\partial S}{\partial y^{i}}
\end{array}\right)=\left(\begin{array}{cc}
g_{i j} & I_{i j} \\
-I_{i j} & g_{i j}
\end{array}\right)\left(\begin{array}{c}
u^{i} \\
v^{i}
\end{array}\right)
$$

If we set $\left(p_{g}\right)_{i}^{x}=g_{i j} u^{i},\left(p_{g}\right)_{i}^{y}=g_{i j} v^{i},\left(p_{I}\right)_{i}^{x}=I_{i j} v^{i},\left(p_{I}\right)_{i}^{y}=I_{i j} u^{i}$, Eq. (14), (15) becomes

$$
\frac{\partial S}{\partial x^{i}}=\left(p_{g}\right)_{i}^{x}+\left(p_{I}\right)_{i}^{x}=(P)_{i}^{x}
$$




$$
\frac{\partial S}{\partial y^{i}}=\left(p_{g}\right)_{i}^{y}-\left(p_{I}\right)_{i}^{y}=(P)_{i}^{y}
$$

and the Hamilton-Jacobi equations will be

$$
P^{i} P_{i}=G^{i j} P_{j} P_{i}=D^{2}
$$

where

$$
P_{i}=\left(\begin{array}{l}
(P)_{i}^{x} \\
(P)_{i}^{y}
\end{array}\right)
$$

It is clear now, that the generalised electromagnetic field $K_{i}$ is nothing else than a momentum defined directly from the symplectic geometry and associated or born from the symplectic form $I_{i j}$ and on the other hand, our common momenta (plus something more as we have seen in the first paper) are defined and associated by the symmetric form $g_{i j}$. But, as we have set $K_{i}^{x}=I_{i j} v^{i}=\left(p_{I}\right)^{x}$ and $K_{i}^{y}=I_{i j} u^{i}=\left(p_{I}\right)^{y}$, nothing can stop us to set $B_{i}^{x}=g_{i j} u^{i}=\left(p_{g}\right)^{x}$ and $B_{i}^{y}=g_{i j} y^{i}=\left(p_{g}\right)^{y}$. As a consequence, the pair Eq. (18), (19) can be written in "field mode" as

$$
\begin{aligned}
& \frac{\partial S}{\partial x^{i}}=\left(B_{i}\right)^{x}+(K i)^{x} \\
& \frac{\partial S}{\partial y^{i}}=\left(B_{i}\right)^{y}-(K i)^{y}
\end{aligned}
$$

This way, the field $B_{i}$ is the unified field with respect to the symmetric form $g_{i j}$ in the same spirit as $K_{i}$ is the unified field with respect with the antisymmetric form $I_{i j}$. Moreover, as we have seen it is related to gravity and dark field! It is important to note that $g_{i j}$ is invariant under rotations of $180^{\circ}$ while $I_{i j}$ is invariant under rotations of $360^{\circ}$. Now, we have the opportunity to separate the two pictures as they are presented. For the first picture, the geometric field is the metric tensor $G_{i j}$, which is separated to the symmetric $g_{i j}$ and antisymmetric $I_{i j}$ geometric fields. This picture is similar to the picture of general relativity or the way we work in general relativity, which is a geometrical theory. The second picture is to work with ordinary fields, a unified one that we will call $\Omega_{i}$, which splits to $B_{i}$ (is produced by $g_{i j}$ ) and $K_{i}$ (is produced by $I_{i j}$ ). These two pictures, are organising the "mess" between existing theories and can provide a concrete framework to work with. A beautiful example as we have mentioned in [?], that shows this "mess" are Kaluza-Klein theories, where we have put the geometrical field $g_{i j}$ together with the field $A_{i}$. As we have shown $g_{i j}$ and $A_{i}$ are incompatible, and that is the reason that Kaluza-Klein theories finally failed to lead us to a unified theory. It is obvious though, that these two pictures are equivalent, it is just a matter of convenience which one we will choose to work with. 


\section{Complex representation}

Next step is to repeat the formalism of the previous section directly to $C^{4}$. The free endpoind will lead us to equations of the form

$$
\frac{\partial S}{\partial z^{i}}=\frac{\partial S}{\partial x^{i}}-i \frac{\partial S}{\partial y^{i}}=G_{i j} U^{i}=P_{i}
$$

where $\mathrm{S}$ is a complex functional of $z: S=S(z)$ due to variation of the free endpoint, $G_{i j}$ is the Hermitian metric tensor, $U^{i}=\frac{d z^{i}}{d s}$ a complex velocity and $P^{i}$ the generalised complex momentum. We can also write this expression as

$$
\frac{\partial S}{\partial z^{i}}=G_{i j} U^{i}=\left(g_{i j}+i I_{i j}\right) U^{i}=g_{i j} U^{i}+i I_{i j} U^{i}
$$

and the conjugate part

$$
\left(\frac{\partial S}{\partial z^{i}}\right)^{*}=\left(G_{i j} U^{i}\right)^{*}==g_{i j}\left(U^{i}\right)^{*}-i I_{i j}\left(U^{i}\right)^{*}
$$

We have again the split of the generalised complex momentum into a momentum defined by $g_{i j}$ and a momentum defined by $I_{i j}$. We can set as previous

$$
\begin{aligned}
& \left(p_{g}\right)_{i}=g_{i j} U^{i}=B_{i} \\
& \left(p_{I}\right)_{i}=I_{i j} U^{i}=K_{i}
\end{aligned}
$$

The physical interpretation remains as it was in the previous section. But, we have the appearance of "i" in front of $K_{i}$, which is of major significance. Let us restrict to the case that $K_{i}$ is just the usual $A_{i}$, Eq. (22) can be read as

$$
\frac{\partial S}{\partial z_{i}}=\left(p_{g}\right)_{i}+i A_{i}=p_{i}^{x}+i p_{i}^{y}+i A_{i}
$$

and let us remove for now the term $p_{i}^{y}$ (which means as we will see that we have remove the mass property), then Eq. (26) becomes 


$$
\frac{\partial S}{\partial x_{i}}+i \frac{\partial S}{\partial y_{i}}=p_{i}^{x}+i A_{i}
$$

the term $p_{i}^{x}$ is the ordinary momentum of the usual 4-d space-time and as a consequence we can set it by p. Moreover, let us again remove the term $\frac{\partial S}{\partial y_{i}}$ and we will have then

$$
\frac{\partial S}{\partial x_{i}}=p+i A_{i}
$$

and if we solve for $p_{i}$

$$
p_{i}=\frac{\partial S}{\partial x_{i}}-i A_{i}
$$

and now let us abstract $\mathrm{S}$ from this expression in order to move into operator form (remember that $\mathrm{S}$ is a complex functional), we will have

$$
\widehat{p_{i}}=\frac{\partial}{\partial x_{i}}-i \widehat{A_{i}}
$$

It is obvious that through our consideration, we have managed to define the usual "covariant derivative" of electromagnetism, which will be better call it Hamilton-Jacodi (H-J) derivative. The only new element is that $A_{i}$, now is an operator and not just a classic field. As a result, for all these years the "covariant derivative" is just the operator $\widehat{p}_{i}$. But, as we have seen, the momentum $p_{i}$ is defined through the metric tensor $g_{i j}$ which is associated to mass, gravity and dark field. By solving the expression Eq. (29) to $p_{i}$, we have lost the information concerning $g_{i j}$, thus we have lost the possibility to describe mass, gravity and dark field. This is why usual quantum theories failed to include gravity and we needed an ad hoc mechanism (Higg's mechanism) to describe the mass property. We must explain ourselves at this point, we do not want to cancel Higg's mechanism or to cancel Standard Model (SM), but rather to reproduce the already known theories in a well defined framework and moreover, to explore new areas of physics. If our consideration is true, then in order to solve only for gravity and dark field, we should solve Eq. (23) with respect to $I_{i j}$ as

$$
P_{i}^{y}=K_{i}=-i \frac{\partial S}{\partial z_{i}}+i B_{i}
$$

or in operator form 


$$
\widehat{P_{i}^{y}}=\widehat{K_{i}}=-i \frac{\partial}{\partial z_{i}}+i \widehat{B_{i}}
$$

Now, if

$$
\left|P_{i}\right|=\left|P^{i}\right|=G_{i j} P_{j} \overline{P_{i}}=G^{i j} \frac{\partial S}{\partial z_{j}}\left(\frac{\partial S}{\partial z_{i}}\right)^{*}=G^{i j} \frac{\partial S}{\partial z_{j}} \frac{\partial S^{*}}{\partial \bar{z}_{i}}
$$

the H-J equations are

$$
G^{i j} \frac{\partial S}{\partial z_{j}} \frac{\partial S^{*}}{\partial \bar{z}_{i}}=D^{2}
$$

where D is some constant. If we subject D to another extremum, we can call as a new Lagrangian

$$
L=D^{2}=G^{i j} \frac{\partial S}{\partial z_{j}} \frac{\partial S^{*}}{\partial \bar{z}_{i}}
$$

and a new action

$$
\mathcal{S}=\int L=\int G^{i j} \frac{\partial S}{\partial z_{j}} \frac{\partial S^{*}}{\partial \bar{z}_{i}}
$$

the extremum will be obtained by varying $\mathcal{S}$

$$
\delta \mathcal{S}=0
$$

and the equations of motion will be

$$
\left(G^{i j} \frac{\partial}{\partial z_{j}} \frac{\partial}{\partial \bar{z}_{i}}\right) \mathcal{S}=0
$$

This equation represents the H-J one in the curved $C^{4}$ space. Let us now write the $L$ in the flat case and see if will remind us something in the existing literature. The flat case for signature $(4,4)$ is just 


$$
L=\frac{\partial \mathcal{S}}{\partial x^{i}} \frac{\partial \mathcal{S}^{*}}{\partial x_{i}}-\frac{\partial \mathcal{S}}{\partial y^{i}} \frac{\partial \mathcal{S}^{*}}{\partial y_{i}}
$$

We can restrict the problem to a subspace of $C^{4}$ by considering that $x_{o}=T$ vanishes. This can happen because as we have mentioned $\mathrm{T}$ is referred to cosmic time, thus we can consider that it changes slowly and will not affect the motion in the subspace. Actually, that should mean also that the scale is fixed in this subspace. Instead of embed spaces, let us try something new by setting

$$
\frac{\partial \mathcal{S}}{\partial y^{i}}=\frac{m}{\hbar} \mathcal{S} \quad \frac{\partial \mathcal{S}^{*}}{\partial y^{i}}=\frac{m^{*}}{\hbar} \mathcal{S}^{*}
$$

where $\mathrm{m}$ is our ordinary mass value and is actually the momentum $p_{y}$ in $\mathrm{Y}$ space (that is the reason that we have called him mass space). This relation suggests that masses are eigenvalues of the operator $\frac{\partial}{\partial y^{i}}$. Eq. (37) becomes

$$
L=\frac{\partial \mathcal{S}}{\partial x^{i}} \frac{\partial \mathcal{S}^{*}}{\partial x^{i}}-\frac{m m^{*}}{\hbar^{2}} \mathcal{S S}^{*}
$$

Now we are ready to make the most surprising observation. If we set

$$
\mathcal{S}=\hbar \varphi
$$

and if $m^{*} \in R$, we have the Klein-Gordon equation for a complex scalar field. Moreover in the case of the flat $G_{i j}$ the antisymmetric part $I_{i j}$ vanishes, which means that there is no "charge". But we have to remember from the first paper [?] that there was a scalar field $E_{i j}$ for which Eq. (36) also holds. We will show later that this scalar is Higg's field. This way we have finally succeed to define as a quantum field related with an elementary field the $\mathcal{S}^{i}=\mathcal{S}\left(z^{i}\right)=\hbar \varphi\left(z^{i}\right)$ which is the solution of Eq. (36). The Lie brackets for this mechanics directly in $C^{4}$ will be

$$
\left[\mathcal{S}_{i}, \mathcal{S}_{j}\right]=0 \quad\left[P_{i}, P_{j}\right]=0 \quad\left[\mathcal{S}_{i}, P_{j}\right]=\delta_{i j}
$$

but if we restrict to the subspace of $C^{4}$ by excluding $\mathrm{T}$ from the equation and replace the eigenvalues the Lie brackets give us the usual Lie brackets of quantum theory! In the next 
paper we will define properly the Lie brackets and their physical meaning. All existing axioms and considerations of the usual quantum theory, are just properties of this treatment. From the waves defined in Y, we observe their momenta, as it happens for every wave. Their momenta is just what we call mass value and this way we have managed to define properly the property of mass and De Broglie's suggestion that all masses have wave properties (matter and wave-particle duality). The probability that is inserted in quantum theories is just our effort to identify a specific point in a wave. We believe that this way the interpretation of quantum theory, now is closed. After all, maybe the hidden variables that many suggested was a reality. The free endpoint that we have used in order to define the particle can be visualised as: imagine an electrified wire where its endpoints are stuck in points a,b, let the endpoint at b, or even both, free and imagine the picture, the electrified wire will start sweeping or scanning all possible positions in space, allowed by its fixed length, trying to find the best path, that through the end-point procedure, still holds! But, this picture happens in the invisible to us space $\mathrm{Y}$, and this behaviour comes from the fact that in $\mathrm{Y}$ we have a usual wave. By releasing the endpoint we have created integrals with an open endpoint, that behave as "path integrals" because it varies along all possible trajectories in space Y. We can repeat all the context of usual mechanics, concerning propagators, for Y space, giving us this way, the opportunity to write actions of the form Wheeler- De Witt, for the space $C^{4}$. Are there many histories this way? We think that we do not need them in the way that they where interpreted till now. We must keep in mind that through all the procedure of the free endpoint the usual geodesic still holds and is fulfilled.

\section{Solving for Higg's field}

The extended equation of energy as it is presented in the first paper [?] is

$$
E^{2}=D^{2} c^{2}+p_{r}^{2} c^{2}-p_{m}^{2} c^{2}+p_{T}^{2} c^{2}
$$

where $p_{r}$ the usual momentum in $R^{3}$ (length space), $p_{m}$ the mass-momentum in $M^{3}$ (mass space), $E_{t}$ the usual energy with respect to $t$ and $p_{T}=E_{T}$ a new type of energy with respect to cosmological time $T=R(t)$ where $\mathrm{R}(\mathrm{t})$ the Cosmos' (Universe) radius and $\mathrm{D}$ a constant with momentum units that must be identified. Let us try to introduce in this equation the constants $c, G, \hbar, G_{F}$. In order to achieve fine tuning in energy scales we introduce the dimensionless constant $\mathrm{A}$, which we call unified constant

$$
A=\frac{1}{6} \sqrt{\frac{2}{3}} \sqrt{\frac{G}{G_{F}}} \frac{h}{c}=3.26297 \times 10^{-18}
$$

where $G_{F}$ is Fermi's constant. The factor

$$
\frac{1}{6} \sqrt{\frac{2}{3}}=\frac{\sqrt{2}}{2} \frac{1}{\sqrt{27}}=\frac{8 \pi}{3} \frac{1}{8} \frac{\sqrt{6}}{6}=8 \pi \frac{1}{24} \frac{\sqrt{6}}{6}
$$


Eq. (40) can be written now as

$$
-\hbar^{2} \frac{\partial^{2} \Psi}{\partial t^{2}}+\hbar^{2} c^{2} \frac{\partial^{2} \Psi}{\partial T^{2}}=D^{2} c^{2}-\hbar^{2} \nabla_{r}^{2} \Psi+A^{2} m_{p}^{4} c^{2} \nabla_{m}^{2} \Psi
$$

if we set

$$
\Psi(\vec{r}, t, \vec{m}, T)=\sigma(t, T) \psi(\vec{r}, \vec{m})
$$

Eq. $\sim 43$ becomes

$$
-\hbar^{2} \frac{1}{\sigma} \frac{\partial^{2} \sigma}{\partial t^{2}}+\frac{1}{\sigma} \hbar^{2} c^{2} \frac{\partial^{2} \sigma}{\partial T^{2}}=D^{2} c^{2}-\frac{1}{\psi} \hbar^{2} \nabla_{r}^{2} \psi+\frac{1}{\psi} A^{2} m_{p}^{4} c^{2} \nabla_{m}^{2} \psi=\omega^{2}
$$

$E q . \sim 45$ splits in two equations as

$$
\begin{gathered}
-\hbar^{2} \frac{\partial^{2} \sigma}{\partial t^{2}}+\hbar^{2} c^{2} \frac{\partial^{2} \sigma}{\partial T^{2}}=\omega^{2} \sigma \\
D^{2} c^{2}-\hbar^{2} \nabla_{r}^{2} \psi+A^{2} m_{p}^{4} c^{2} \nabla_{m}^{2} \psi=\omega^{2} \psi
\end{gathered}
$$

we split $\sigma(t, T)=\varphi(t) g(T)$ and $E q . \sim 47$ becomes

$$
c^{2} \frac{1}{g} \frac{\partial^{2} g}{\partial T^{2}}-\frac{1}{\varphi} \frac{\partial^{2} \varphi}{\partial t^{2}}=\left(\frac{\omega}{\hbar}\right)^{2}
$$

and if we call $\frac{1}{\varphi} \frac{\partial^{2} \varphi}{\partial t^{2}}=k^{2}$

$$
\frac{1}{g} \frac{\partial^{2} g}{\partial T^{2}}=\left(\left(\frac{\omega}{\hbar c}\right)^{2}+\left(\frac{k}{c}\right)^{2}\right)
$$

and if we call $\rho^{2}=\left(\left(\frac{\omega}{\hbar c}\right)^{2}+\left(\frac{k}{c}\right)^{2}\right)$

$$
\frac{1}{g} \frac{\partial^{2} g}{\partial T^{2}}=\rho^{2}
$$


so we get two equations

$$
\begin{aligned}
& \frac{1}{\varphi} \frac{\partial^{2} \varphi}{\partial t^{2}}=k^{2} \\
& \frac{1}{g} \frac{\partial^{2} g}{\partial T^{2}}=\rho^{2}
\end{aligned}
$$

with general solutions $\varphi(t)=a_{1} e^{-k t}+a_{2} e^{k t}$ and $g(t)=b_{1} e^{-\rho T}+b_{2} e^{\rho T}$. If we restrict the solutions to $t, T \geq 0$ we have

$$
\sigma(t, T)=N e^{-\rho T} e^{k t}
$$

where $\mathrm{N}$ constant. Moreover $e^{-\rho T}$ can be normalised if we set $\int_{0}^{\infty} N^{2} e^{2 \rho t} d T=1$ which means $N=\sqrt{2 \rho}$. Furthermore we can calculate the mean value of $\mathrm{T}$ as

$$
<T>_{t}=\int_{0}^{\infty} T\left(\sqrt{2 \rho} e^{-\rho T} e^{k t}\right)^{2}=\frac{1}{2 \rho} e^{2 k t}=\frac{1}{2 \sqrt{\left(\left(\frac{\omega}{\hbar c}\right)^{2}+\left(\frac{k}{c}\right)^{2}\right)}} e^{2 k t}
$$

$E q . \sim 54$ resembles the equation of the universe of De-Sitter in a cosmos with vacuum domination

$$
R(t)=\frac{\sqrt{2}}{2} \frac{c}{H} e^{H t}
$$

On the other hand the time independent $E q . \sim 47$ splits in two equations if we set

$$
\begin{gathered}
\psi(\vec{r}, \vec{m})=\zeta(\vec{r}) \xi(\vec{m}) \\
D^{2} c^{2}-\frac{1}{\zeta} \hbar^{2} \nabla_{r}^{2} \zeta=\mu^{2} \\
\omega^{2}-\frac{1}{\xi} A^{2} m_{p}^{4} c^{2} \nabla_{m}^{2} \xi=\mu^{2}
\end{gathered}
$$


we will solve $E q . \sim 57$ using spherical coordinates $(r, \theta, \phi)$. If we set $\zeta(r, \theta, \phi)=R(r) \Upsilon_{1}(\theta, \phi)$, where $\Upsilon_{1}(\theta, \phi)$ our usual spherical harmonic functions and $q^{2}=\left(\frac{\mu^{2}-D^{2} c^{2}}{\hbar^{2} c^{2}}\right) r^{2}$ and $\lambda_{1}=$ $\frac{1}{\Upsilon_{1}} L_{r}^{2} \Upsilon_{1}$ where $L_{r}$ the angular-momentum operator $E q . \sim 58$ becomes

$$
q^{2} \frac{d R}{d \phi^{2}}+2 q \frac{d R}{d q}+\left(q^{2}-\lambda_{1}\right) R=0
$$

which is a Bessel's differential equation. As a conclusion, the solution of Eq. 57 for a spherical infinite well of radius $r_{0}$ becomes

$$
\mu^{2}-D^{2} c^{2}=\frac{q_{l_{1}, k}^{2} \hbar^{2} c^{2}}{r_{0}^{2}}
$$

where $q_{l, k}$ the roots of Bessel's function. This solution in the case that $l_{1}=0$, the roots are $q_{0, k}=k \pi$ for $k=1,2, \ldots$. On the other hand the equation $E q . \sim 58$ is mathematically equivalent. As a result we can set spherical coordinates $(m, \Theta, \Phi)$. If we set $\xi(m, \Theta, \Phi)=$ $M(m) \Upsilon_{2}(\Theta, \Phi)$, where $\Upsilon_{2}(\Theta, \Phi)$ our usual spherical harmonic functions in $M^{3}$ and $Q^{2}=$ $\left(\frac{\mu^{2}-\omega^{2}}{A^{2} m_{P}^{4} c^{2}}\right) m^{2}$ and $\lambda_{1}=\frac{1}{\Upsilon_{1}} L_{r}^{2} \Upsilon_{1}$ where $L_{m}$ the angular-momentum operator in mass-space $M^{3}$ the equation becomes as

$$
Q^{2} \frac{d M}{d \Phi^{2}}+2 Q \frac{d M}{d Q}+\left(q^{2}-\lambda_{2}\right) M=0
$$

where $\lambda_{2}=l_{2}\left(l_{2}+1\right)$. The solution in the same spirit is

$$
\mu^{2}-\omega^{2}=\frac{q_{l_{2}, k}^{2} A^{2} m_{p}^{4} c^{2}}{m_{0}^{2}}
$$

where $m_{0}$ is the radius in mass-space. For the case $l_{2}=0$ the roots are $q_{0, k}=k \pi$ for $k=1,2, \ldots$ and the solution becomes

$$
\mu^{2}-\omega^{2}=\left(\frac{k \pi A^{2} m_{P}^{2} c^{2}}{m_{0}}\right)^{2}
$$

Afterwards if we consider that $m_{0}=m_{p}$ we can have 


$$
\sqrt{\mu^{2}-\omega^{2}}=k \pi A m_{P} c^{2}
$$

in addition, if we set $k=1$ which is the ground state $(k \neq 0$ from the solution of the differential equation) we have a mass eigenvalue

$$
m=\pi A m_{P}=125,173945 G e v / c^{2}
$$

Moreover, if we set equivalently $l_{0}=l_{P}$ in $E q . \sim 60$ we can have

$$
\sqrt{\mu^{2}-D^{2} c^{2}}=k \pi m_{P} c^{2}
$$

from the comparison of Eq. 64 with $E q . \sim 66$ we can get

$$
\omega=A D c=E_{H}
$$

Moreover the above mentioned assumption combined with $E q . \sim 27$ explains the transformation $y^{\alpha^{\prime}}=\lambda \delta_{\varrho}^{\alpha^{\prime}} x^{\varrho}$ for $\alpha^{\prime}=1,2,3$ and $y^{0}=y^{0}\left(x^{0}\right)$ as it was proposed in our first paper. We have to mention that this transformation is now a prediction of our original consideration and must be connected with the scales $k, \lambda$ of the first paper.

\section{Remarks from the solution}

1. We have seen that $<R(t)>_{t}=<T>_{t}=\frac{1}{2 \rho} e^{2 k t}$ give us an exponentially behaviour for $\mathrm{T}=\mathrm{R}(\mathrm{T})$. If we want to see the nature of $\mathrm{t}$, we must write

$$
\ln <T>_{t}=\ln \left(\frac{1}{2 \rho} e^{2 k t}\right)
$$

and if we solve with respect to $t$

$$
t=\frac{\ln <T>+\ln \varrho}{2 k}
$$

It is obvious, that $\mathrm{t}$ and $\mathrm{T}$ are in different scale and of different nature and function, as it was originally hypothesised. Moreover, if we examine the solution 


$$
\Psi(\vec{r}, t, \vec{m}, T)=\Psi_{r} \Psi_{m} e^{-k t} e^{\rho T}
$$

we can figure that $t$ decreases exponentially while $\mathrm{T}$ increase exponentially. This assumption gives us in some sense that $\mathrm{t}$ is a "local" time, while $\mathrm{T}$ is a global one. This effect seems natural if we remember that proper time $\tau$ is combined with the time of a mass body propagating in the usual space-time. The only problem that seems to exist, is that a particle described by a wave function has the form in the usual space-time

$$
\Psi(\vec{r}, t)=\Psi_{r} e^{-i k t}
$$

while in the expression described by equation $E q . \sim 70$, the time depended term has the form $e^{-k t}$. But in the first paper we have note that

$" m_{0}\left(m_{0} \in R\right)$ describes a mass moving in the usual space-time originating from the sub-space $M^{3}$. Different subspaces of $R^{7}$ express different $m_{1}, m_{2}, \ldots$. that move inside different subspaces of the usual space-time, forming different " cosmic lines" for different masses $m_{i}$, which are connected through usual Lorentz transformations."

Thus, if we consider that local measurements do not be affected by the motion of Cosmos or that $\Delta T \rightarrow 0$, we can ignore the parameter $T$. This way, if we look back the equation $E q . \sim 46$, the solution becomes for the time depended part $e^{-i k t}$ as it should be for a particle propagating in the usual space-time. Moreover, we can see that there is a big difference under local measurements $(\mathrm{t})$ and global measurements $(\mathrm{T})$. In order to compromise the different measurements we need to abstract the information through the logarithm. This behaviour could explain the problem of the cosmological constant where the theoretical expectation value of vacuum (quantum mechanics) is 54 orders of magnitude bigger than the measured one.

2. The mass value of Higg's boson as was mentioned is

$$
m=\pi A m_{P}=\frac{1}{6} \sqrt{\frac{2}{3}} \pi \sqrt{\frac{\hbar^{3}}{G_{F} c}}
$$

where we can see that finally its mass value is independent by G. Moreover we have to mention that we have not included any corrections such as Darwin correction due to spin 0. That means that the final value would be little less than $125,173945 \mathrm{Gev} / \mathrm{c}^{2}$.

3. As concerned the constant A, we could avoid to include by the beginning. But as is well accepted, a Klein-Gordon equation describes particles with spin 0. Moreover, as it was presented, we expected that the extended Klein-Gordon equation, which was derived directly from the geometry of the flat $R^{8} \simeq C^{4}$, should be linked with the vacuum i.e the Higg's boson (in the case of spherically infinite well). As a conclusion, we have seen that a dimensionless constant with value around $10^{-17}$ should be added. Due to this observation, combined with the empirical Fermi's relation about $G_{F}$, we suspected that this constant should be the above mentioned constant A. Moreover, it looks attractive 
the fact that the four basic constants in Physics $\left(G_{F} \mathrm{~g}, \hbar, \mathrm{c}\right)$ are unified in a single dimensionless form. As concerned the factor $=\frac{1}{6} \sqrt{\frac{2}{3}}$, it was derived by the expression of the general (curved) metric tensor $G \in G L(4, C)$ in the Gell-Mann basis, where the factor $\frac{1}{12} \frac{\sqrt{6}}{6}$ has appeared in the $G_{44}$ component. Afterwards, we have suspected that it could be derived directly from the number of generators of $S O(4,4)$. Thus, the factor could have the form

$$
\frac{\sqrt{2}}{2} \sqrt{\frac{1}{n-1}}
$$

where $\mathrm{n}$ the number of the generators. An interesting fact, that should be interpreted in the future, is that this factor appears in the literature of black holes' horizon, if someone started from the relation of the density and set $R=r_{g}$

$$
\begin{gathered}
d=\frac{M}{V}=\frac{3 M}{4 \pi R^{3}} \rightarrow R=\left(\frac{3 M}{4 \pi d}\right)^{\frac{1}{3}} \\
R=r_{g} \rightarrow \frac{1}{d}=\frac{32 \pi}{3} \frac{G^{3}}{c^{6}} M^{2}=\left(24 \frac{1}{6} \sqrt{\frac{2}{3}}\right)^{2} \pi \frac{G^{3}}{c^{6}} M^{2}
\end{gathered}
$$

Additionally, we can examine which is the scale involved with the constant A

$$
\ln \frac{1}{A}=-\ln A=40,263894 \rightarrow\left(\ln \frac{1}{A}\right)^{-1}=\frac{1}{40,263894}
$$

while the pure constant $A^{\prime}$ (without the factor) is

$$
\ln \frac{1}{A^{\prime}}=\frac{1}{38,2694}
$$

At this part, we imagine that the constant A, should be interpreted as initial condition of Cosmos. In that way, Cosmos could begin by only one Higg's boson (excitation of vacuum), where this boson under tunnelling effect raised to the step $E_{P}$. The most crucial element of the model, are the Planck units or Planck epoch. Specifficaly, the Planck units indicates that they serve as a critical point for the model. The Planck units can be derived under the hypothesis that the Swhartchild's radius $r_{g}$ is equal to Compton's wavelength $\left(l_{C}\right)$. If we consider that Cosmos had been in that state (Planck epoch), we can see that exactly after this epoch, we have a disconnection of $r_{g}$ and $l_{c}$ and they present two different physical phenomena. An alternative scenario in order to explain the jump from Higg's vacuum to Planck energy avoiding tunnelling, 
is to hypothesize that an external cause made that jump to exactly Planck energy. Eventually, whatever scenario one chooses to propose or to accept, it seems that Cosmos seems to drain energy all the time by a "storage" unit, which is succeeded through "local" time t to "global" time T.

4. Let us consider two different states of Cosmos which for some reasons, are interesting in order to examine. State $\mathrm{A}$ where Cosmos has energy, radius and mass $\left(E_{1}, T_{1}=\right.$ $\left.R_{1}(t), M_{1}\right)$ and state $\mathrm{B}$ with $\left(E_{2}, T_{2}, M_{2}\right)$ respectively. If we write the formulas about $T_{1}, T_{2}$

$$
\begin{aligned}
& <R_{1}(t)>=T_{1}=\frac{c}{H_{1}(t)} e^{H_{1}(t) t_{1}} \\
& <R_{2}(t)>=T_{2}=\frac{c}{H_{2}(t)} e^{H_{2}(t) t_{2}}
\end{aligned}
$$

from these equations we can find also

$$
H_{2} t_{2}-H_{1} t_{1}=\ln \left(\frac{H_{2} t_{2}}{H_{1} t_{1}}\right)
$$

We can observe that the quantities $H_{i} t_{i}$ are dimensionless. On the other hand, in the context of high energy physics, we have the renormalisation group equations that lead to

$$
\frac{1}{a_{i}\left(Q^{2}\right)}-\frac{1}{a_{i}\left(\mu^{2}\right)}=\frac{b_{i}}{4 \pi} \ln \left(\frac{Q^{2}}{\mu^{2}}\right)
$$

where $\mathrm{Q}$ the mass energy scale which we use in order to measure the coupling constants $a_{i}$ and $\mu$ the scale in which we measure the coupling constant and $b_{i}$ coefficients that come from the groups of invariance. Even if we examined only the flat case, while as we have seen the electromagnetic, nuclear and strong nuclear fields will appear in the curved space, we can assume that equations $E q . \sim 80,83$ are equivalent. The difference lies only in the presentation. In cosmology we are mostly interesting in the physical quantities of radius and Hubble's constant while in high energy physics in the energy scales. Thus high energy physics and cosmology are equivalent sectors of physics.

5. We can think the manifold $C^{4}$ as a $C^{3}$ manifold consisting of vectors $\vec{r}, \vec{m}$ and a $C$ manifold consisting of t and T. Afterwards, the "time" manifold $C$ is embedded in the $C^{3}$ where the vectors becomes $\vec{r}(t, T)$ and $\vec{m}(t, T)$. Meanwhile, let us consider a "sphere" where the bundle is the $C^{3}$ plus the time t (forming a 7 -d manifold), while the time $\mathrm{T}$ is the radius of this "sphere". The evolutionary behaviour of $\mathrm{T}$ makes this "sphere" to expand. The inner "vacuum" which acts as a "trompe" reflects on the expansion of the bundle. In the beginning $t$ and $\mathrm{T}$ are comparable, but after Planck's epoch $\mathrm{t}$ and $\mathrm{T}$ follow different scale. 
6. It is important to note that, vacuum as a real zero (really empty space) has no meaning at all. An actual zero, should mean that we do not have solutions for the H-J equation. Even in flat space, there is geodesic equation, which gives us a H-J equation, which has a solution $\mathcal{S}=\hbar \varphi$, which has at least an eigenvalue. Moreover, the symplectic $R^{8}$ is locally flat, which suggests that we always find the flat metric tensor to work with and that we always find the Higg's field there to wait as the minimum case of a scalar field theory. We can not avoid the Higg's field.

\section{Symmetries in curved $C^{4}$}

The H-J equation Eq. (36) must be the unified theory of fields in the curved space $C^{4}$, where curved means that all possible fields are included in this expression. We will try to investigate what happens if we embed the usual space-time $R^{4}$ in the curved $C^{4}$ using the language of groups, which is more familiar to those that are investigating grand-unified theories. The group of the relation $G_{i j}=g_{i j}+i I_{i j}$ comes as

$$
G L(4, C)=S O(4,4) \cap U(4)
$$

This group after we embed will come as

$$
G L(1,3, R) \times \frac{G L(4, C)}{G L(1,3, R)}=\left(S O(1,3) \times \frac{S O(4,4)}{S O(1,3, R)}\right) \cap\left(S U(2) \times \frac{S U(4)}{S U(2)} \times U(1)\right)
$$

From our usual 4-d space-time we "see" the $S O(1,3)$ for Minkowski metric tensor of special relativity and $S U(2)$ But, $S U(2)$ is only locally isomorphic to $S O(1,3)$ which suggests that we have a local symmetry. This will effect all fields that come from the $I_{i j}$ form. Everything, except these two symmetries comes as an external cause. This is why we have to add additional symmetries in all our efforts of a grand unified theory. The breaking of the original symmetry which is dictated by $E q . \sim 82$ to the symmetry dictated by $E q . \sim 83$ is exactly what we call spontaneous symmetry breaking and it represents our effort to observe the $C^{4}$ from the usual $R^{4}$ space. It is interesting to observe that the $\frac{S U(4)}{S U(2)}$ in the last parenthesis of $E q . \sim 83$ has 12 generators. On the other hand, the group $G=U(1) \times S U(2) \times S U(3)$ of standard model has exactly 12 generators, as well. At this point, we have to wonder ourselves if $\mathrm{G}$ representation provide us with a complete unified theory for electromagnetism, weak nuclear field and strong nuclear field. As a matter of fact, the strong nuclear field was never really unified in the sense that electromagnetism and weak nuclear field were, due to the fact that the $S U(3)$ symmetry was intact and never broken because it successfully gave us many of the properties that we needed in order to describe the strong nuclear field. But, it failed to describe the mass gap problem and quark confinement, as well. Our hypothesis is either the group 


$$
\frac{S U(4)}{S U(2)}
$$

has the same algebra with the group $G=U(1) \times S U(2) \times S U(3)$ but they are not isomorphical as groups, either the group described by $E q . \sim 86$ can be the original symmetry of strong nuclear field, that finally reduces to the unbroken symmetry of $S U(3)$ that describes colour. Specifically, in the second case, there is an operator $\mathrm{T}$ such that

$$
T\left(\frac{S U(4)}{S U(2)}\right) \longrightarrow S U(3)
$$

but we have to investigate the properties of such a group transformation and see what will be the consequences and if it is consistent with the standard model. The picture, not by symmetries, but from fields directly, is for $g_{i j}$ we have a field $g_{i j} u^{i}$, for $D_{i j}$ we have a field $D_{i j} u^{i}$, for $E_{i j}$ we have a scalar field $E_{i j} u^{i}$ and from $M_{i j}$ we have a field $M_{i j} u^{i}$. If we replace this fields in Eq. $\sim 36$ we will have all the interactions between the fields and Eq. $\sim 38$ will represent the equation of motion of those fields. All the above mentioned analysis represents only bosons. That way, we have

$$
\begin{gathered}
g_{i j} u^{i}=G r_{j} \longrightarrow \text { graviton } \\
D_{i j} u^{i}=D_{j} \longrightarrow \text { dark boson } \\
M_{i j} u^{i}=A_{j}, W_{j}, G_{j} \longrightarrow \text { photon, weak bosons, gluons } \\
E_{i j} u^{i}=H_{j}=H \longrightarrow \text { Higg's boson }
\end{gathered}
$$

With the benefit of the doubt, if we proceed the same way as we do in the context of standard model, for the group $S O(4,4)$, we could find the number of bosons of gravity and dark field. The $S O(4,4)$ breaks as we have seen in the first paper to $S O(1,3), S O(3,1)$ and to a symplectic group which has 16 real generators. If we keep $S O(1,3)$ for the standard $g_{i j}$ and company $S O(3,1)$ for the other part of $g_{i j}$ which is multiplied by the scale, locally we have the isomorphy to $S U(2)$, which leads us to the assumption that there exist 3 gravitons. On the other hand, if the symplectic group can locally be isomorphical to $S U(3)$, there must exist 8 dark bosons and the Higg's boson is left with the displacements. Another way to interpretate what $S O(4,4)$ stands for is to consider that in the beginning we have a unified Higg's field (not scalar) $\mathrm{H}(\mathrm{z})$ that breaks into three fields as 


$$
H(z) \longrightarrow H_{g}+H_{D}+H_{E}
$$

where $H_{g}(z)$ is a "Higg's field" connected to gravity, $H_{D}(z)$ is a "Higg's field" connected to dark field and $H_{E}(z)$ is the usual Higg's scalar field. These three different fields have three different energy scales and properties. The physics underlying the part $S O(4,4)$ is still mysterious and under consideration. But we must proceed and conclude with the properties of the "field" $\mathcal{S}=\hbar \varphi$ which satisfies Eq. (39) or Eq. (40). This "field" $\mathcal{S}$ must be invariant under transformations of the group $G L(4, C)$ as Eq. (36) suggests. This group is a Lie complex one, of complex dimension $n^{2}$, connected but not simple, with its maximal compact Lie subgroup is $U(n)$ and has a fundamental group isomorphic to $\mathcal{Z}$. Moreover, we can write

$$
\mathcal{S} \in G L(4, C) \longrightarrow S O(4,4) \cap U(4)
$$

which means that $\mathcal{S}$ is invariant under transformations both of the extended Lorentz group $S 0(4,4)$ and $U(4)$. After the embedding, the original group breaks further and must be invariant under transformations imposed by Eq. (85). The cap $\cap$ suggests that the generators of the group will be correlated or mixed, combining this way all the generators. At this point, we must remember that in the standard formulation of the standard model, we must start by a symmetry group imposed directly in the covariant derivative, further we introduce a symmetry for the field $\varphi$ (i.e Higg's $S U() 2)$ doublet) and afterwards we seek the transformation rule for the fields $A_{\mu}, W_{\mu}, G_{\mu}$. In our consideration, we have by the beginning the transformation rule for the "field" $\mathcal{S}=\hbar \varphi$ and afterwards we seek how $\frac{\partial \mathcal{S}}{\partial z}$ transforms, which will tell us about the invariance of the Lagrangian. In the case that we want to re-introduce the standard model we must solve Eq. (23) with respect to $p_{g}$ as

$$
\widehat{p_{i}}=\frac{\partial}{\partial x_{i}}-i \widehat{K_{i}}
$$

and then seek the invariance of the quantity

$$
\widehat{p_{i}} \mathcal{S}=\frac{\partial}{\partial x_{i}} \mathcal{S}-i \widehat{K_{i}} \mathcal{S}
$$

But, by solving with respect to $p_{g}$, we will lose the information of Lorentz invariance and we will left only with $U(4)$ invariance. This way we should add by hand the Lorentz invariance. The same picture, comes with the formulation of the standard model, where we impose both Lorentz and $U(1), S U(2), S U(3)$ invariance, plus the ad-hoc addition of Higg's field. The Lagrangian described by Eq. (35) is also invariant under transformations of the group $G L(4, C)$. Now, if $\lambda_{i}$ are the generators of $G L(4, C)$, the $\lambda_{i}$ coincides with the generators 
of $U(4) \simeq S U(4) \times U(1)$ (the difference between $U(4)$ and $G L(4, C)$ is the different factors, in the case of $U(4)$ the factors are all real, while in the case of $G L(4, C)$ are all complex ). Moreover, $G L(4, C)$ locally falls naturally to $U(4)$ as the maximal compact subgroup and therefore we can write for $\mathcal{S}$

$$
\mathcal{S}^{\prime} \longrightarrow \mathcal{S}^{\prime}=U \mathcal{S}=e^{i \theta_{\alpha} H_{\alpha}} \mathcal{S}
$$

where $\theta_{\alpha}$ the parameters and $H_{\alpha}$ the generators of the group and $\alpha=1, \ldots . .16$. For infinitesimal parameters $\left|\theta_{\alpha}\right|<<1$ we can have

$$
\begin{gathered}
\mathcal{S}^{\prime}(z) \simeq\left(I_{16}+i \theta_{\alpha} H_{\alpha}\right) \mathcal{S}(z) \\
\delta \mathcal{S}(z)=i \theta_{\alpha} H_{\alpha} \in \mathcal{S}(z) \\
\delta \mathcal{S}^{*}(z)=-i \theta_{\alpha} H_{\alpha} \in \mathcal{S}(z)
\end{gathered}
$$

But, after embedding $R^{4}$ in $C^{4}$, the group $G L(4, C)$ will break according to Eq. (85) and each group as it is presented in Eq. (85), will be accompanied by a different scale, as it is was originally indicated in our first paper [?]. Moreover, the original generators of $G L(4, C)$, will break into different generators of the broken groups that are presented in Eq. (85). An extensive study on Eq. (85), and if there are no mistakes in our treatment of Eq. (85), should be able to provide us, with an extension of standard model, which could be the desired unified theory, by the point of view of symmetry group study. But until now, we have "managed" to describe what it happens only in the case of bosons. Fermions will be derived in the fifth paper [?] not only by the usual Dirac method, but from Cartan's property of triality and afterwards directly from the geometry by considering 1-linear forms. In our next paper [?], we will proceed by varying the endpoint in the case of the action

$$
S_{\pi}=\int Z \sqrt{g} d \Omega
$$

\section{Conclusion}

We have shown that usual quantum theories are contained in the classic mechanics theory in $C^{4}$, by identifying the complex functional action, as the scalar field $\varphi$ and afterwards in the curved case, we have derived the covariant derivative of gauge theories, as a natural extension of the 4-d complex energy-momentum vector. Interactions of the form $B_{\mu \nu} B^{\mu \nu}, W_{\mu \nu} W^{\mu \nu}$.., will be investigated in the fourth paper [?] of this series. 


\section{References}

\section{References}

[1] D. Mastoridis, K. Kalogirou, $C^{4}$ Space-Time.. a window to new Physics, www.researchgate.net.

[2] D. Mastoridis, K. Kalogirou, Field Equations in $C^{4}$ space-time, www.researchgate.net.

[3] D. Mastoridis, K. Kalogirou, Introduction to Quantum Field Theory in $C^{4}$ space-time, www.researchgate.net.

[4] D. Mastoridis, K. Kalogirou, Dynamic Path in $C^{4}$ space-time, www.researchgate.net.

[5] D. Mastoridis, K. Kalogirou, Introduction to Fermionic Structures in $C^{4}$ space-time, www.researchgate.net. 